\title{
A hierarchical facility layout planning approach for large and complex hospitals
}

\author{
Stefan Helber, Daniel Böhme, Farid Oucherif, \\ Svenja Lagershausen, and Steffen Kasper \\ Department of \\ Production Management \\ Leibniz University Hannover, Germany \\ stefan.helber@prod.uni-hannover.de, +49 5117625650 \\ March 3, 2014
}

\begin{abstract}
The transportation processes for patients, personnel, and material in large and complex maximum-care hospitals with many departments can consume significant resources and thus induce substantial logistics costs. These costs are largely determined by the allocation of the different departments and wards in possibly multiple connected hospital buildings. We develop a hierarchical layout planning approach based on an analysis of organizational and operational data from the Hannover Medical School, a large and complex university hospital in Hannover, Germany. The purpose of this approach is to propose locations for departments and wards for a given system of buildings such that the consumption of resources due to those transportation processes is minimized. We apply the approach to this real-world organizational and operational dataset as well as to a fictitious hospital building and analyze the algorithmic behavior and resulting layout.
\end{abstract}

Keywords: Hospital layout planning, quadratic assignment problem, fix-and-optimize heuristic 


\section{Introduction}

Large hospitals often consist of many highly specialized departments with corresponding wards. The allocation of these organizational units (OUs) within the hospital buildings determines the distances and travel times between those OUs. In the daily operation of a hospital, patients, medical personnel, and material must travel or be transported between these OUs. The resource consumption due to travel and transportation processes depends on the chosen location of the different facilities within the hospital.

Allocating those facilities is a highly complex problem that is rarely solved in a formal and systematic approach. It is also a challenging problem from a mathematical perspective, as it contains the quadratic assignment problem (QAP), which is difficult to solve to optimality. In addition, a substantial number of important real-world constraints and problem aspects must be considered to develop a formal hospital facility layout planning approach.

To develop such an approach, we decompose the problem in a hierarchical manner into two planning stages. In the Stage I problem, we assign OUs to locations considering various factors, such as space requirements. Based on this assignment, we then make more detailed decisions regarding the internal location of the selected OUs within those locations in the Stage II problem. Despite the hierarchical problem decomposition, the Stage I problem is still numerically difficult to solve via standard solvers for mixedinteger programs. Thus, we also present a fix-and-optimize heuristic decomposition approach to solve the Stage I problem. We then apply the developed facility layout planning system to a real-world dataset of departments, wards, and travel intensities. We consider a fictitious system of connected hospital buildings, for which distances can be computed in a systematic and transparent manner. For this system of buildings, we determine an allocation of the OUs to minimize the consumption of resources due to transportation processes.

The remainder of the paper is structured as follows. In Section 2, we present the problem in more detail and discuss the related literature. The hierarchical modeling approach is explained in Section 3. Section 4 contains the fix-and-optimize heuristic for the Stage I problem. The case study for the real-world organizational dataset is presented in Section 5, and conclusions are provided in Section 6. 


\section{The hospital facility layout problem (HFLP)}

\subsection{Problem aspects}

When hospitals are designed, extended, or re-organized, the locations of different OUs within the hospital building(s) must be determined. This is a complex task for large maximum-care hospitals with numerous highly specialized departments and sub-departments. This task determines the spatial location of the numerous medical departments, such as

- Accident and emergency

- Anesthesia

- Diagnostic imaging

- General surgery

- Gynecology

- Oncology

as well as the location of other (non-medical) service units. Many of the medical departments have corresponding wards, where the patients stay until they are ready to leave the hospital. In a large hospital, more than 1,000 beds can be distributed over those wards. Due to their size and high degree of specialization, such large hospitals often also serve as teaching hospitals to train future physicians or are even part of, or associated with, a university. In this case, such hospitals also provide research and teaching facilities as well as numerous administrative and social facilities.

The spatial distances between the different departments and between departments and wards can be significant in these large hospitals. A substantial staff can be necessary in the hospital's transportation service to transport patients. Furthermore, transports of numerous types of materials, such as beds, food, and medical supplies, are also necessary. Transporting patients can range from guiding people (who might otherwise become lost) to pushing wheelchairs or beds. As the fraction of patients with extreme obesity increases, an increasing fraction of transport tasks require two members of the transportation service to transport a bed with a single obese patient. In addition, the medical staff (physicians and nurses) can spend an economically relevant part of their working time merely traveling from one location to another within the hospital. For 


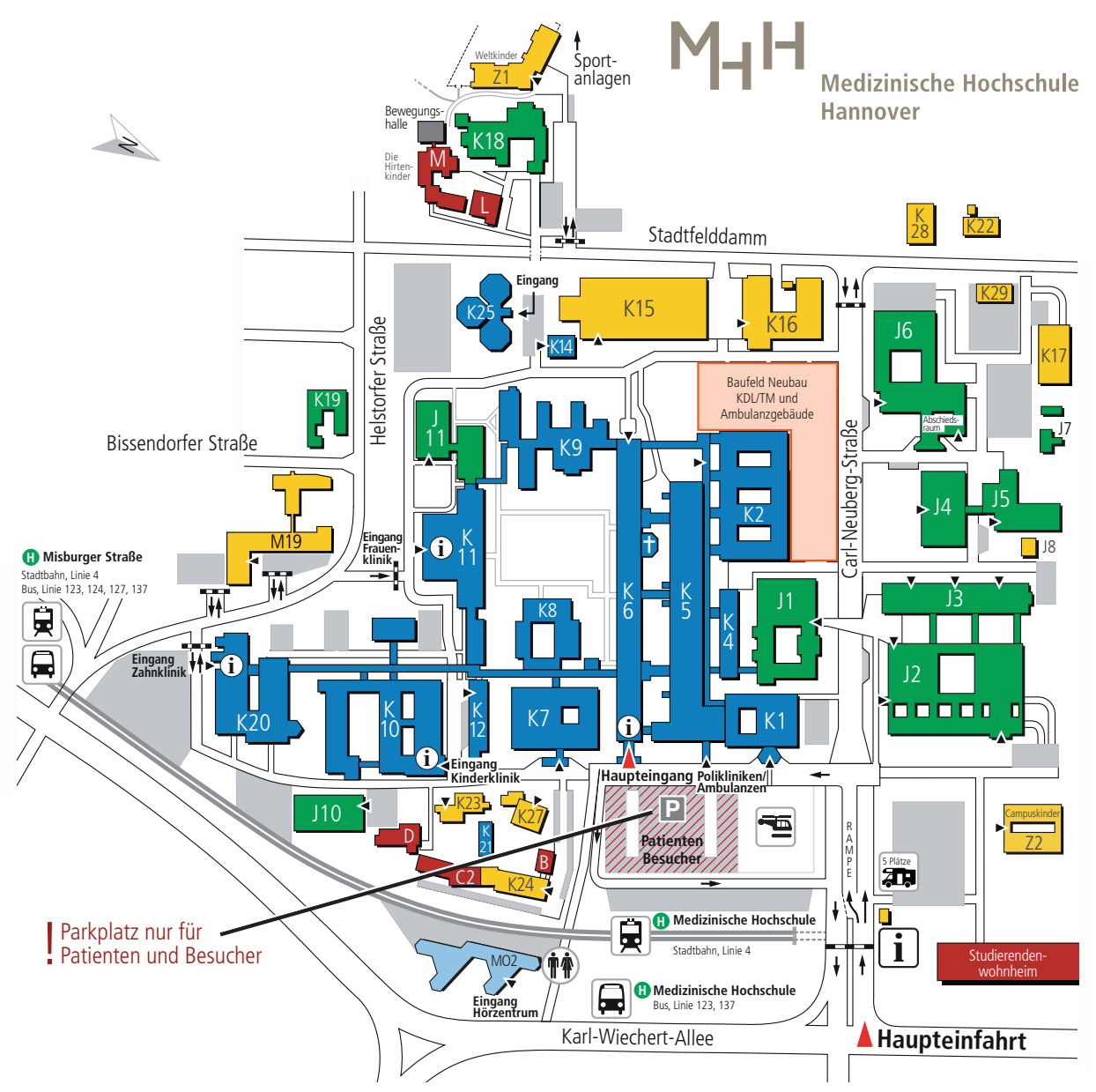

Figure 1: Site plan of the Hannover Medical School, Source: Medizinische Hochschule Hannover (MHH)

those reasons, the highly heterogenous transportation processes are a major concern in hospital facility layout planning.

As a practical example regarding organizational and operational aspects, we consider the case of the MHH, one of Germany's leading medical schools. Figure 1 presents the spatial dimensions and complexity of this maximum-care hospital. A computerized dispatching system for transportation jobs was installed at the MHH several years ago. The archive of those past transportation jobs can be used to determine the transportation intensity between different OUs, such as functional departments or wards. In Oucherif (2012, p. 7), almost 630,000 records of patient transportation jobs for the 12-month period from May 2011 to April 2012 were analyzed to determine those transportation intensities. In addition, data on the currently used space of the different OUs were collected in Oucherif (2012). This real-world dataset is useful for highlighting the organizational and operational aspects of the HFLP due to its size and complexity. 
This paper does not seek to reoptimize the current MHH layout. If the MHH buildings were to be rebuilt today given the MHH's current scale and scope, a more compact and logistically efficient structure of the buildings would most likely be chosen. Instead, we address the general HFLP at a more abstract level and develop a decision support system. However, to evaluate this system, we use the MHH dataset to create a test bed by combining it with a fictitious system of buildings.

A decision support system for hospital layout planning must consider the following factors:

- Organizational units differ with respect to their space requirements. To the extent possible, they should be locally concentrated in a compact manner to minimize internal transportation processes.

- Some OUs may require particular locations or locations that have a specific relevant feature. For example, the Emergency Department or Emergency Room should be accessible by ambulances, and thus, an allocation at ground level is typically necessary. Similarly, specific technical requirements may enforce or eliminate certain allocations of OUs to locations.

- There may be considerations that prohibit a direct neighborhood of two specific units; for instance, patients that recently underwent a surgical procedure should be protected from infectious areas. In contrast, there may be cases where a direct neighborhood is highly desirable for operational purposes. For example, medical personnel may have to move routinely back and forth between a specific medical department's functional installations and the department's wards.

We address all these features in the model system presented below. One might argue that this facility layout problem cannot be solved in reality, as the transportation data will only be available if the hospital has already been in operation for some time, i.e., after all the facility layout decisions have been made. Although this is generally true, it is possible to establish standardized datasets for transportation processes of different types of hospitals. Analyzing a dataset for a hospital with a similar organizational structure to obtain an estimate of the future transportation volume may still be helpful for determining reasonable layout proposals for a new or redesigned hospital.

\subsection{Related literature}

In the research literature, the general facility layout problem is mainly discussed with respect to manufacturing systems. However, the related methodology is highly relevant 
for the allocation of departments in hospitals. Drira et al. (2007) have recently conducted a literature review on facility layout problems. The authors classify the existing literature according to numerous criteria, such as a static versus dynamic problem setting, continuous or discrete decision variables, and the type of problem formulation. Levary and Kalchik (1985) summarize the main characteristics of the most frequently used solution procedures. Kusiak and Heragu (1987) present and compare different formulations of the facility layout problem and corresponding algorithms to solve the presented problem. Hassan (1994) have conducted a literature review focused on the machine layout problem.

Facility layout problems typically contain a QAP. Loiola et al. (2007) have surveyed the literature on the QAP. The authors present selected QAP formulations and discuss theoretical resources used to define lower bounds for exact and heuristic algorithms. Burkard et al. (1991) summarize the main QAPs and establish a library of problem instances for the QAP. Many studies on the QAP are related to fields other than the hospital layout problem. For instance, Steinberg (1961) addresses the problem of placing computer elements on the mainboard, Hillier and Connors (1966) examine the problem of assigning locations to indivisible facilities and its relation to the QAP, and Burkard and Offermann (1977) consider the allocation of keys on a computer keyboard. Many heuristics have been proposed for the QAP because the QAP is one of the most difficult problems in the NP-hard class and has a wide area of applications. Hahn et al. (2010) and Hahn et al. (2012) used the reformulation-linearization technique to solve QAP. Additional methods have been proposed by Glover and Woolsey (1974), Armour and Buffa (1963), Foulds (1983), Adams and Sherali (1986), and Oral and Kettani (1992). Heragu and Kusiak (1991) present two linearizations for the facility layout problem. Bozer et al. (1994) develop an approach to consider facilities with multiple floors by extending an existing facility layout algorithm. Bozer and Meller (1997) compare basic modeling approaches for the facility layout problem. Barbosa-Póvoa et al. (2001) propose a mixed-integer linear model to find the optimal two-dimensional layout. In a subsequent study, Barbosa-Póvoa et al. (2002) extend their model to consider a threedimensional space.

A relatively limited body of research has been published with respect to layout planning in hospitals. Elshafei (1977) formulates the problem of locating hospital departments as a QAP to minimize the total distance traveled by patients. Hahn and Krarup (2001) outline the history of a facility layout problem that arose in 1972 when designing another large German Hospital, Klinikum Regensburg. The problem was formulated as a QAP, solved in 1972, and included in the QAP library of Burkard et al. (1991). Butler 
et al. (1992) consider the facility layout and bed allocation problems in a two-stage approach. In the first stage, an optimization model for the facility layout is solved, and in the second stage, a simulation-optimization procedure for bed allocation is performed. Vos et al. (2007) evaluate a hospital building design with the focus on ensuring that the design supports the efficient and effective operating of care processes. The authors evaluate different scenarios in a discrete-event simulation. Hahn et al. (2010) formulate the multi-story space assignment problem, which is particularly relevant in hospital layout planning. However, we are not aware of a hospital facility layout planning approach that can handle large and heterogeneous dataset as well as the numerous real-world aspects of the problem, as reported in Oucherif (2012).

\section{A hierarchical modeling approach}

\subsection{Conceptual starting point: The QAP}

To develop a formal decision support system for the HFLP, we assume that a planned or existing building or system of buildings is given. Within these buildings, spatial areas that will house OUs must be identified. In our context, OUs are in particular medical departments with their functional installations and wards. The spatial areas are divided into locations. The distances between different locations $k$ and $l$ are denoted as $d_{k l}$. Similarly, transport processes with a number $m_{i j}$ of transports per time unit occur between different OUs $i$ and $j$ (in either direction). It is sufficient to consider transport relations with $i<j$ because the direction of the traffic is not relevant.

A natural starting point to formally model the HFLP is the QAP (see Koopmans and Beckmann (1957)). The QAP aims at assigning OUs $i, j \in \mathscr{I}$ to locations $k, l \in \mathscr{K}$ such that each OU is assigned to exactly one location, whereas each location houses at most one OU, and the total transport cost is minimized. The central decision variable $X_{i k}$ equals 1 if $\mathrm{OU} i$ is assigned to location $k$ and 0 otherwise. The QAP can be stated as follows:

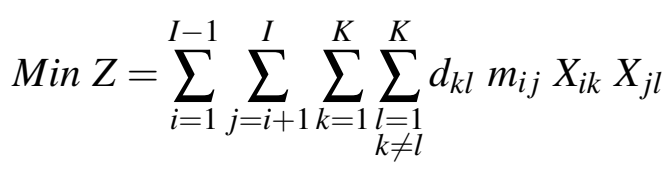




$$
\begin{aligned}
& \text { s.t. } \\
& \sum_{i=1}^{I} X_{i k} \leq 1 \quad k \in \mathscr{K} \\
& \sum_{k=1}^{K} X_{i k}=1 \quad i \in \mathscr{I} \\
& X_{i k} \in\{0,1\} \quad i, j \in \mathscr{I} ; k, l \in \mathscr{K}
\end{aligned}
$$

The QAP is a quadratic problem due to the product $X_{i k} \cdot X_{j l}$ of decision variables in the objective function. It can be linearized by introducing a new variable $\widetilde{X}_{i k j l}$. This binary variable equals 1 if OU $i$ is assigned to location $k$ and OU $j$ is assigned to location $l$. It substitutes the product $X_{i k} \cdot X_{j l}$ in the objective function, which subsequently becomes

$$
\operatorname{Min} Z=\sum_{i=1}^{I-1} \sum_{j=i+1}^{I} \sum_{\substack { k=1 \\
\begin{subarray}{c}{l=1 \\
k \neq l{ k = 1 \\
\begin{subarray} { c } { l = 1 \\
k \neq l } }\end{subarray}}^{K} d_{k l} m_{i j} \widetilde{X}_{i k j l}
$$

The following additional constraint ties the $X_{i k}$ variables to the $\widetilde{X}_{i k j l}$ variables:

$$
X_{i k}+X_{j l}-\widetilde{X}_{i k j l} \leq 1 \quad i, j \in \mathscr{I} ; i<j ; k, l \in \mathscr{K} ; k \neq l
$$

With this linearization, a standard mixed-integer linear programming (MILP) solver can theoretically solve the QAP. However, the problem is still difficult to solve for even small instances. The number of variables $\widetilde{X}_{i k j l}$ and the number of constraints (6) rapidly become prohibitively large when addressing even relatively small problem instances. In addition, the QAP does not begin to approach the real-world complexities of allocating the OUs of a hospital. However, the QAP is a useful conceptual starting point to formally consider those complexities in a hierarchical problem decomposition.

\subsection{Hierarchical problem decomposition}

Large maximum-care hospitals are often distributed over various buildings, each of which may have several levels. Thus, the distances between two locations $k$ and $l$ have both a horizontal and vertical component $\left(d_{k l}^{H}\right.$ and $d_{k l}^{V}$, respectively). These components are defined by floors and the location of elevators or staircases, as illustrated in Figure 2. The distances reflect the structure of the given or planned building(s) and are exogenously given in our modeling perspective.

In the basic version of the QAP presented in Section 3.1, we assume that each location can theoretically house each of the OUs and that each OU is assigned to a maximum 


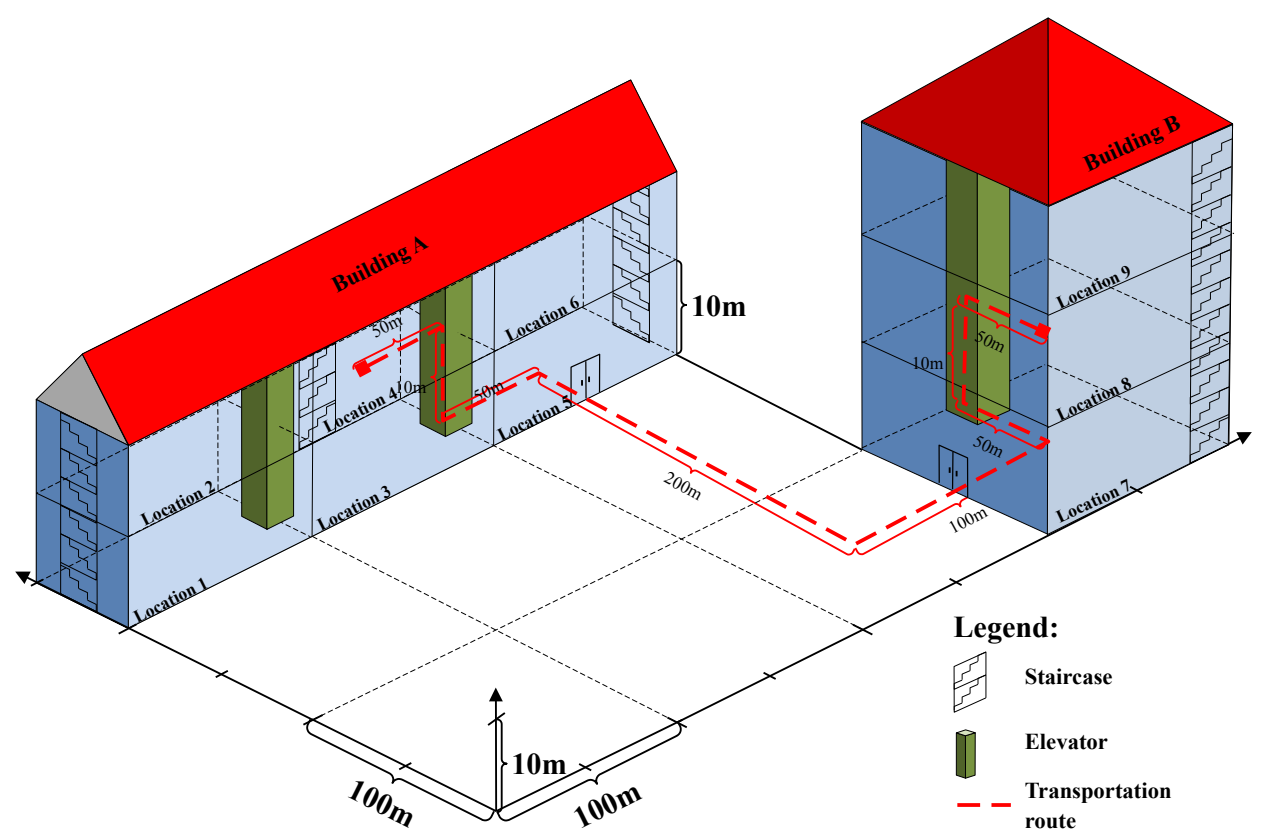

Figure 2: Distances and transportation routes

of one location. In reality, the space requirements of the different OUs of a hospital can differ by a factor of 10, i.e., a relatively small fraction of the OUs can account for a relatively large fraction of the total space requirement. Thus, a large OU may require multiple (possibly adjacent) locations, whereas other locations may house multiple small OUs. Thus, we assume that an OU $i \in \mathscr{I}$ can consist of multiple OU elements $e \in \mathscr{E}_{i}$. The space requirement $a_{i e}$ for such an OU element $e$ of OU $i$ should not exceed the size of those locations $\mathscr{K}_{i}$ that are candidates to house the respective OU. The analysis in Oucherif (2012) for the MMH indicates that a large number of the OUs are relatively small with respect to their space requirements in relation to the available space at the locations. For each of those OUs, only one such OU element $e$ needs to be defined because a single location suffices to house that OU.

Furthermore, the transportation tasks differ considerably with respect to the tolerable transportation time. For non-critical transport types, such as the transport of used beds, a long transportation time due to a large distance only leads to high transportation costs for the required personnel. However, a long travel time between, for instance, the helicopter landing pad and cardiology department may be extremely undesirable. Consider the case of transporting an urgent patient with a myocardial infarction ("heart attack"). For this type of transport, there may be a maximum tolerable travel time. Due to this reason, different types $b$ of transport with maximum travel times $t_{b}^{\max }$ must be defined. The different transport types $b$ may also differ with respect to the horizon- 


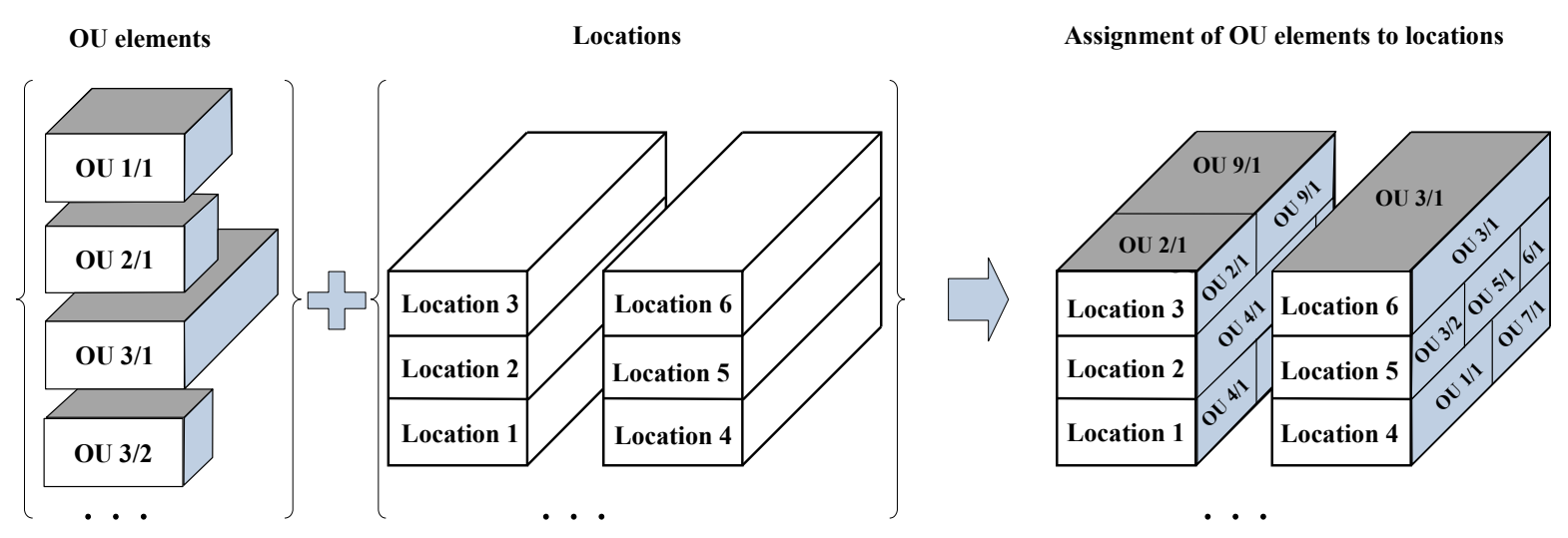

Figure 3: Assignment of OU elements to locations in Stage I

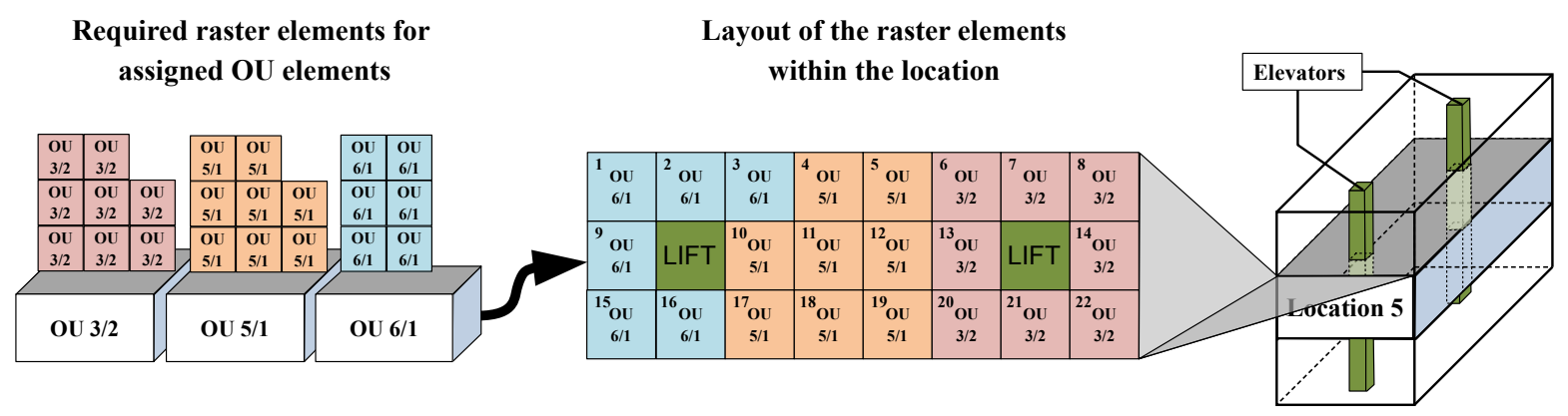

Figure 4: Location of required raster elements for assigned OU elements in Stage II

tal and vertical transportation speeds $\left(v_{b}^{H}\right.$ and $v_{b}^{V}$, respectively). These are examples of real-world complexities that must be reflected in a potentially useful decision support system, in addition to the problem aspects already outlined in Section 2.1. These aspects complicate the HFLP from both managerial and mathematical perspectives.

Thus, we developed a two-stage decision support system based on the principles of aggregation and decomposition to reduce the conceptual complexity and numerical burden of proposing a layout.

We assumed that each of the OUs to be allocated represents an aggregation of objects that are similar in function and structure and that have tight internal organizational ties. For example, several wards belonging to the same medical department may be aggregated to a single care area. This aggregation reduces the number of OUs that have to be considered. Furthermore, we assume that a location is a larger spatial area, such as the entire floor of a larger building. Each of these locations could house multiple OUs. Thus, this aggregation approach limits both the number of OUs and locations to be considered.

Figures 3 and 4 illustrate the concept of the proposed approach. In the Stage I 
problem treated in Figure 3, we assign the elements $e \in \mathscr{E}_{i}$ of all the OUs $i \in \mathscr{I}$ to the locations $k \in \mathscr{K}$. For example, OU 3 consists of two elements, $3 / 1$ and $3 / 2$, which are assigned to locations 6 and 5 , respectively. Whereas OU element $3 / 1$ is sufficiently large that it requires an entire floor of the right building, OU element $3 / 2$ is substantially smaller and can hence share a floor with OU elements $5 / 1$ and $6 / 1$ in location 5 . This Stage I problem contains, among other components, a QAP. Due to the reduced number of aggregate objects, solving this problem is demanding but feasible. In addition to or as a variation of the QAP, we make the following additional assumptions for the Stage I problem:

- A location has a spatial capacity constraint and can host multiple OU elements.

- There may be upper limits on the desired travel time for a given type of transport.

- If an OU consists of multiple elements, these elements should be assigned to immediately adjacent locations.

- It should be possible to impose a limit on the total dispersion of multi-element OUs over different locations.

The Stage II problem then considers the Stage I planning results and treats each location separately. The aim of this stage is to place the possibly multiple OU elements assigned to a location within that location. To explain the approach, we consider the case of location 5, to which the OU elements $3 / 2,5 / 1$, and 6/1 were assigned, in Figure 4. We assume that the spatial area of each location can be divided into rectangular raster elements, which may reflect the technical construction of the building. The space requirement for the possibly multiple elements $e$ of OU $i$ that are assigned to the given location is converted into the number $q_{i}$ of raster elements required for those elements of OU $i$. In Figure 4, eight raster elements are required for OU elements $3 / 2$ and 5/1, whereas six raster elements are required for OU element 6/1. In the Stage II problem, these required raster elements must be placed within the currently considered location 5. In this problem, we aim to cohesively place the raster elements within each location. Furthermore, the OUs that need to use elevators most often should be placed close to the elevators. These OUs exhibit high traffic intensity to and from OUs at other locations. The following two sections describe the models for both stages in detail. The models contain elements developed in Böhme (2013). 
Table 1: Notation for the Stage I model

\begin{tabular}{|c|c|}
\hline \multicolumn{2}{|c|}{ Indices and sets } \\
\hline$b \in \mathscr{B}$ & Transport types $b=1, \ldots, B$ \\
\hline$i, j \in \mathscr{I}$ & Organizational units (OU) $i, j=1, \ldots, I$ \\
\hline$e, f \in \mathscr{E}_{i}$ & Elements of OU $i$ \\
\hline$k, l \in \mathscr{K}$ & Locations $k, l=1, \ldots, K$ \\
\hline $\mathscr{K}_{i} \subseteq \mathscr{K}$ & Locations that can house the elements of OU $i$ \\
\hline $\mathscr{N}_{k} \subseteq \mathscr{K}$ & Locations that are immediate neighbors of location $k$ \\
\hline \multicolumn{2}{|c|}{ Parameters } \\
\hline$a_{i e}$ & Space requirement of element $e$ of $\mathrm{OU} i$ \\
\hline$A_{k}$ & Available space at location $k$ \\
\hline$d_{k l}^{H}, d_{k l}^{V}$ & Horizontal/vertical distance between locations $k$ and $l$ \\
\hline$d c_{k}^{H}, d c_{k}^{V}$ & $\begin{array}{l}\text { Horizontal/vertical distance between location } k \text { and the spatial } \\
\text { center of the hospital }\end{array}$ \\
\hline$G$ & $\begin{array}{l}\text { Maximum aggregated number of additional locations used by } \\
\text { multi-element OUs }\end{array}$ \\
\hline$m_{i e j f b}$ & $\begin{array}{l}\text { Number of transports between element } e \text { of OU } i \\
\text { and element } f \text { of OU } j \text { for transport type } b\end{array}$ \\
\hline $\operatorname{Loc}_{i j}^{+}$ & $=\left\{\begin{array}{l}1 \text { if } \mathrm{OE} i \text { and } \mathrm{OE} j \text { must be assigned to the same location } \\
0 \text { otherwise }\end{array}\right.$ \\
\hline $\operatorname{Loc}_{i j}^{-}$ & $=\left\{\begin{array}{l}1 \text { if } \mathrm{OE} i \text { and } \mathrm{OE} j \text { cannot be assigned to the same location } \\
0 \text { otherwise }\end{array}\right.$ \\
\hline$p c_{b}$ & Penalty "cost" for exceeding the time limit for transport type $b$ \\
\hline$t_{b_{x}}^{\max }$ & Maximum tolerable transport time for transport type $b$ \\
\hline$v_{b}^{H}$ & Horizontal transport speed of transport type $b$ \\
\hline$v_{b}^{V}$ & Vertical transport speed of transport type $b$ \\
\hline \multicolumn{2}{|c|}{ Variables } \\
\hline$X_{i e k}^{I}$ & $=\left\{\begin{array}{l}1 \text { if element } e \text { of OU } i \text { is assigned to location } k \\
0 \text { otherwise }\end{array}\right.$ \\
\hline$\widetilde{X}_{i e k j f l}^{I}$ & $=\left\{\begin{array}{l}1 \text { if element } e \text { of } \mathrm{OE} i \text { is assigned to location } k \text { and } \\
\text { element } f \text { of OU } j \text { to location } l \\
0 \text { otherwise }\end{array}\right.$ \\
\hline$U_{\text {iekjflb }}$ & Exceedance of the travel time limit \\
\hline$Y_{i k}$ & $=\left\{\begin{array}{l}1 \text { if any element of OU } i \text { is assigned to location } k \\
0 \text { otherwise }\end{array}\right.$ \\
\hline$W_{i}$ & Number of locations housing at least one element of OU $i$ \\
\hline$Z^{I}$ & Stage I objective function value (total transport workload) \\
\hline
\end{tabular}




\subsection{Stage I: Assigning elements of OUs to locations}

With the notation given in Table 1, we now define the Stage I model as follows:

$$
\text { Min. } Z=\sum_{i=1}^{I-1} \sum_{e \in \mathscr{E}_{i}} \sum_{k \in \mathscr{K}_{i}} \sum_{j=i+1}^{I} \sum_{f \in \mathscr{E}_{j}} \sum_{\substack{l \in \mathscr{K}_{j} \\ l \neq k}} \sum_{b=1}^{B} m_{i e j f b}\left(\left(\frac{d_{k l}^{H}}{v_{b}^{H}}+\frac{d_{k l}^{V}}{v_{b}^{V}}\right) \widetilde{X}_{i e k j f l}^{I}+p c_{b} U_{\text {iekjflb }}\right)
$$

subject to

$$
\begin{array}{ll}
\sum_{i=1}^{I} \sum_{e \in \mathscr{E}_{i}} a_{i e} X_{i e k}^{I} \leq A_{k} & k \in \mathscr{K} \\
\sum_{k \in \mathscr{K}_{i}} X_{i e k}^{I}=1 & i \in \mathscr{I}, e \in \mathscr{E}_{i} \\
\left(\frac{d_{k l}^{H}}{v_{b}^{H}}+\frac{d_{k l}^{V}}{v_{b}^{V}}\right) \widetilde{X}_{i e k j f l}^{I} \leq t_{b}^{m a x}+U_{i e k j f l b} & i, j \in \mathscr{I}, i<j, e \in \mathscr{E}_{i}, k \in \mathscr{K}_{i}, f \in \mathscr{E}_{j}, l \in \mathscr{K}_{j}, \\
X_{i e k}^{I}=X_{j f k}^{I} & k \neq l, b \in \mathscr{B} \\
X_{i e k}^{I}+X_{j f k}^{I} \leq 1 & i, j \in \mathscr{I}, k \in \mathscr{K}_{i}, L o c_{i j}^{+}=1, e \in \mathscr{E}_{i}, f \in \mathscr{E}_{j} \\
X_{i e k}^{I}+X_{j f l}^{I}-\widetilde{X}_{i e k j f l}^{I} \leq 1 & i, j \in \mathscr{I}, k \in \mathscr{K}_{i}, L o c_{i j}^{-}=1, e \in \mathscr{E}_{i}, f \in \mathscr{E}_{j} \\
X_{i e k}^{I} \leq X_{i, e+1, k}^{I}+\sum_{l \in \mathscr{N}_{k} \cap \mathscr{K}_{i}} X_{i, e+1, l}^{I} & i, j \in \mathscr{I}, i<j, k \in \mathscr{K}_{i}, l \in \mathscr{K}_{j}, k \neq l, \\
\sum_{e \in \mathscr{E}_{i}} X_{i e k}^{I} \leq\left|\mathscr{E}_{i}\right| \cdot Y_{i k} & i \in \mathscr{I}, e \in \mathscr{E}_{i}, e<\left|\mathscr{E}_{i}\right|, k \in \mathscr{K}_{i} \\
\sum_{k \in \mathscr{K}_{i}} Y_{i k}=W_{i} & i \in \mathscr{I},\left|\mathscr{E}_{i}\right|>1, k \in \mathscr{K}_{i} \\
\sum_{i \in \mathscr{I}}\left(W_{i}-1\right) \leq G & i \in \mathscr{I} \\
\left|\mathscr{E}_{i}\right|>1 & i \in \mathscr{I},\left|\mathscr{E}_{i}\right|>1 \\
X_{i e k}^{I}, \tilde{X}_{i e k j f l}, Y_{i k} \in\{0,1\} & \\
W_{i} \in\{0,1,2, \ldots\} &
\end{array}
$$

The objective function (7) aims to minimize the total transportation time considering both the distances between locations and the number of transports between OUs. It further penalizes any exceedance of the travel time limit. Constraint (8) is the spatial capacity constraint for the locations. Constraint (9) is used to assign each element $e \in \mathscr{E}_{i}$ of OU $i$ to one location out of the set $\mathscr{K}_{i}$ that can house that OU. Any exceedance of the maximum tolerable travel time is detected in constraint (10). When a pair $(i, j)$ 
of OUs must be assigned to the same location, this requirement is enforced for all respective elements $e$ and $f$ via constraint (11). Similarly, constraint (12) prohibits the joint assignment of the elements of two incompatible OUs to the same location. In constraint (13), the linearization is performed as in (5). The use of the decision variable

$\widetilde{X}_{i k j l}$ induces symmetry in the model because $\widetilde{X}_{i k j l}=1$ describes the same decision as $\widetilde{X}_{j l i k}=1$. Thus, the definition domain in constraints (10) and (13) and the summation in objective function (7) can be restricted to pairs $(i, j)$ with $i<j$. If an OU $i$ consists of multiple elements $e$, constraint (14) ensures that those elements are assigned to either the same location $i$ or an immediate neighbor location $l \in \mathscr{N}_{k}$. This constraint enforces a connected placement of the possibly multiple elements of an OU. Constraints (15) and (16) determine the number of locations occupied by multi-element OUs. Constraint (17) limits the extent to which the elements of multi-element OUs are spread over multiple locations.

Because the Stage I problem contains a computationally challenging QAP, we describe a heuristic math programming algorithm to solve the problem below.

\subsection{Stage II: Detailed positioning within a location}

The model for the Stage II problem is solved for each location $k$ separately. The location is divided into uniformly sized raster elements $r \in \mathscr{R}^{k}$. The space requirement for all elements $e \in \mathscr{E}_{i}$ of OU $i \in \mathscr{I}^{k}$ assigned to location $k$ is expressed in terms of the number of raster elements $q_{i}$ needed for elements of OU $i$ that must be placed within that location. Two often conflicting objectives are considered in the model. We want to place those OUs that have heavy traffic to and from other locations $l \neq k$ close to the elevators. This preference is controlled via a binary assignment variable $X_{i r}^{I I}$, which indicates whether raster element $r$ is used by an element of OU $i$. However, we also want to achieve compact allocations of those raster elements used by a particular OU $i \in \mathscr{I}^{k}$. To this end, we use the integer variable $Y_{i r}$, which denotes the number of raster elements immediately next to raster element $r$ that house the same OU $i$. Maximizing this quantity induces a compact allocation of the OUs within the currently considered location. Using the notation shown in Table 2, detailed positions for OUs within the locations can be found via the following model:

$$
\text { Minimize } Z^{I I}=\alpha \sum_{i \in \mathscr{I}^{k}} \sum_{r \in \mathscr{R}^{k}} \frac{m_{i}^{L i f t} d_{r}^{L i f t}}{q_{i}} X_{i r}^{I I}-\sum_{i \in \mathscr{I}^{k}} \sum_{r \in \mathscr{R}^{k}} Y_{i r}
$$


Table 2: Notation of the Stage II model for location $k$

\section{Indices and sets}

$i \in \mathscr{I}^{k}$

$r, p \in \mathscr{R}^{k}$

$\mathscr{N}_{r} \subseteq \mathscr{R}^{k}$

Organizational units $i$ with elements $e \in \mathscr{E}_{i}$ assigned to location $k$ Raster elements of location $k$

Set of raster elements that are immediate neighbors of raster element $r$

\section{Parameters}

$\alpha$

$d_{r}^{L i f t}$

$m_{i}^{\text {Lift }}$

$q_{i}$

\section{Variables}

$X_{i r}^{I I}$

$Y_{i r}$

$Z^{I I}$
Weight and normalization factor

Distance between raster element $r$ and the nearest elevator

Number of transports between OU $i$ and OUs at other locations (and hence via the elevator)

Number of raster elements required for OU $i \in \mathscr{I}^{k}$

subject to

$$
\begin{array}{ll}
Y_{i r} \leq q_{i} \cdot X_{i r}^{I I} & i \in \mathscr{I}^{k}, r \in \mathscr{R}^{k} \\
Y_{i r} \leq \sum_{p \in \mathscr{N}_{r}} X_{i p}^{I I} & i \in \mathscr{I}^{k}, r \in \mathscr{R}^{k} \\
\sum_{r \in \mathscr{R}^{k}} X_{i r}^{I I}=q_{i} & i \in \mathscr{I}^{k} \\
\sum_{i \in \mathscr{I}^{k}} X_{i r}^{I I} \leq 1 & r \in \mathscr{R}^{k} \\
X_{i r}^{I I} \in\{0,1\}, Y_{i r} \in\{0,1,2,3, \ldots\} & i \in \mathscr{I}^{k}, r \in \mathscr{R}^{k}
\end{array}
$$

In objective function (20), the transportation effort to the elevator(s) is minimized and the internal compactness of the set of raster elements assigned to OU $i$ is maximized via the sum of the neighborhood relationships. The parameter $\alpha$ serves two purposes. First, it harmonizes the different dimensions of the two components of the objective function. Second, it balances the compactness of the solution against the transportation effort to the elevator(s). Constraint (21) allows for positive values of the variable $Y_{i r}$ 
only if OU $i$ uses raster element $r$. The number of raster elements in the immediate neighborhood of raster element $r$ that are also used by OU $i$ is considered in constraint (22) through the optimization direction of the objective function. Each OU $i$ seizes the required number of $q_{i}$ raster elements using equation (23). Constraint (24) prohibits the assignment of more than one OU to each raster element $r$.

The Stage II problem can easily be solved for each location $k$ in isolation using standard mixed-integer linear programming software. The solution may be subject to manual fine-tuning by human planers considering additional aspects that are not considered in the Stage II model.

\section{A fix-and-optimize heuristic for the Stage I problem}

\subsection{Finding an initial feasible solution}

The Stage I problem presented in Section 3.3 is a computationally challenging NP-hard optimization problem because it includes the QAP as a special case (see Garey and Johnson (2009, p. 218)). We developed an iterative improvement heuristic to solve the problem. Below, we first describe two different approaches to construct an initial solution. We then demonstrate how this initial solution can be improved iteratively.

The Stage I problem is difficult due the "quadratic component" reflected in the possibly large number of $\widetilde{X}_{i e k j f l}$ variables. Thus, we construct the initial solution by avoiding this variable and optimize over the $X_{i e k}$ variables only, which also forces us to omit constraints (10) and (13) temporarily. In other words, we start by solving a relatively straightforward assignment problem. The two different methods of constructing a feasible starting solution differ slightly with respect to the objective function. The first approach (denoted as approach "A") that appears to suggest itself is to place the elements of heavy-traffic OUs close to the spatial center of the layout and the remaining low-traffic OUs somewhere in the periphery. This solution can be modeled very easily based on the horizontal and vertical distances of location $k$ to this spatial center of the hospitals ( $d c_{k}^{H}$ and $d c_{k}^{V}$, respectively) using the following objective function:

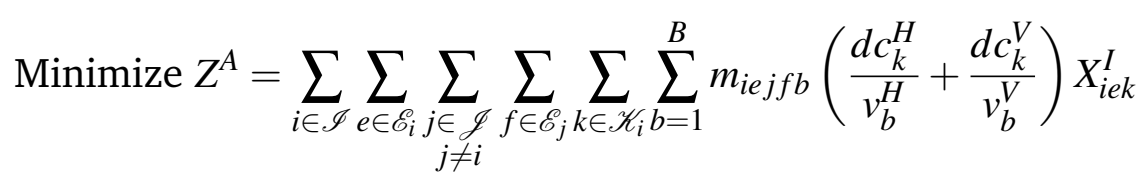

The objective function (26) is linear. Minimizing this function subject to restrictions (8), (9), (11), (12), and (14) to (19) is less computationally demanding than solving the 
original Stage I model presented in Section 3.3. This solution leads to a first feasible assignment of OUs $i$ to locations. For this or any other solution, the non-zero values of the $\widetilde{X}_{i e k j f l}$ variables of the original Stage I model can be easily calculated from the given values of the $X_{i e k}^{I}$ variables. Furthermore, any exceedance $U_{\text {iekjflb }}$ of the travel time limit $t_{b}^{\max }$ can be easily determined via constraint (10). Hence, one can easily determine the objective function value of the original Stage I model for the starting solution. A second approach "B" to find a starting solution is to use the same weight for all decision variables $X_{i e k}^{I}$ according to the following modified objective function:

$$
\text { Minimize } Z^{B}=\sum_{i \in \mathscr{I}} \sum_{\substack{e \in \mathscr{E}_{i} \\ j}} \sum_{\substack{j \in \mathscr{J} \\ j \neq i}} \sum_{f \in \mathscr{E}_{j}} \sum_{k \in \mathscr{K}_{i}} \sum_{b=1}^{B} X_{i e k}^{I}
$$

In preliminary tests, approach $\mathrm{A}$, with the objective function (26), led to a better initial solution than approach B, with the objective function (27), for the initial (QAP-type) Stage I problem. Furthermore, use of the initial solution from approach B can also be more time consuming. However, this solution tends to lead to better final solutions, so we used and compared both approaches below. Regardless of which approach is used, the result is an initial set of values for the current (incumbent) solution. This set is stored as the parameter $X S_{i e k}^{I}$, i.e., we perform an initial assignment

$$
X S_{i e k}^{I}:=X_{i e k}^{I}, \quad i \in \mathscr{I}, e \in \mathscr{E}_{i}, k \in \mathscr{K}_{i}
$$

to remember the current solution.

\subsection{Iterative improvement of the feasible solutions}

We use an iterative fix-and-optimize heuristic decomposition to improve the incumbent solution (see Sahling (2010) or Helber and Sahling (2010)). In this approach, we first split the set of OUs $\mathscr{I}$ into two subsets $\mathscr{I}^{\text {opt }}$ and $\mathscr{I}^{\text {fix }}=\mathscr{I} \backslash \mathscr{I}^{\text {opt }}$. The set $\mathscr{I}^{\text {fix }}$ denotes all OUs for which the allocation of their elements $e \in \mathscr{E}_{i}$ remains fixed to their $X S_{i e k}^{I}$ values in the current Stage I subproblem. Hence, only the location of elements of OUs within the set $\mathscr{I}^{\text {opt }}$ can be optimized. Similarly, we split the set of locations $\mathscr{K}$ into two subsets, $\mathscr{K}^{\text {opt }}$ and $\mathscr{K}^{\text {fix }}=\mathscr{K} \backslash \mathscr{K}^{\text {opt }}$. To this end, we also add the following two constraints to the Stage I model as formulated in Section 3.3:

$$
\begin{array}{ll}
X_{i e k}^{I}=X S_{i e k}^{I} & i \in \mathscr{I}^{\mathrm{fix}}, e \in \mathscr{E}_{i}, k \in \mathscr{K}_{i} \\
X_{\text {iek }}^{I}=X S_{\text {iek }}^{I} & i \in \mathscr{I}, e \in \mathscr{E}_{i}, k \in \mathscr{K}^{\text {fix }}
\end{array}
$$


$/ *$ Part I: Determine an initial feasible solution */

Maximize (26) or (27) subject to (8), (9), (11), (12), (14) - (19)

Set $X S_{i e k}^{I}:=X_{i e k}^{I}$ to store incumbent solution

Determine initial objective function value $Z^{I, \text { best }}$ via (7) and (10) for the incumbent solution

$/ *$ Part II: Improve feasible solutions via partial optimization */

Set RoundsWithoutImprovement $:=0$

while RoundsWithoutImprovement $<$ LimitRoundsWithoutImprovement and TimeLimitNotExceeded do

RoundsWithoutImprovement $:=$ RoundsWithoutImprovement +1

$/ *$ Optimize over the random selection of OUs and all locations */

Determine the random set $\mathscr{I}^{\text {opt }} \subseteq \mathscr{I}$ with \#OU ${ }^{\text {opt }}$ elements

Set $\mathscr{I}^{\text {fix }}:=\mathscr{I} \backslash \mathscr{I}^{\mathrm{opt}}$

$\mathscr{K}^{\text {fix }}:=\{\}$

Solve the augmented Stage I model (7) - (19), (29), and (30)

if $Z^{I}<Z^{I, b e s t}$ then

Set $X S_{i e k}^{I}:=X_{\text {iek }}^{I}$ to store the improved solution

Set RoundsWithout Improvement $:=0$

Set $Z^{I, \text { best }}:=Z^{I}$

end

$/ *$ Optimize over the random selection of locations and all OUs */

Determine the random set $\mathscr{K}^{\text {opt }} \subseteq \mathscr{K}$ with \#Loc ${ }^{\text {opt }}$ elements

Set $\mathscr{K}^{\text {fix }}:=\mathscr{K} \backslash \mathscr{K}^{\text {opt }}$

$\mathscr{I}^{\text {fix }}:=\{\}$

Solve the augmented Stage I model (7) - (19), (29) and (30)

if $Z^{I}<Z^{I, b e s t}$ then

Set $X S_{i e k}^{I}:=X_{i e k}^{I}$ to store the improved solution

Set RoundsWithout Improvement $:=0$

Set $Z^{I, \text { best }}:=Z^{I}$

end

end

Algorithm 1: Fix-and-optimize algorithm for the Stage I problem 
After finding an initial solution, as described in Section 4.1, we first set $\mathscr{K}^{\mathrm{fix}}:=\{\}$ and determine a random set $\mathscr{I}^{\text {opt }}$ and a corresponding set $\mathscr{I}^{\text {fix }}$. In the next step, we solve the Stage I problem augmented by constraints (29) and (30), which may lead to an improved solution. We then optimize over the locations. To this end, we set $\mathscr{I}^{\text {fix }}:=\{\}$ and determine a random set $\mathscr{K}^{\text {opt }}$ as well as a corresponding set $\mathscr{K}^{\text {fix }}$. We again solve the Stage I problem augmented by constraints (29) and (30). The process is repeated until no improvement is obtained over a specified number of consecutive iterations. The number of $\widetilde{X}_{i e k j f l}$ variables in each of the augmented Stage I problems is reduced considerably by adding constraints (29) and (30). Any solution to the augmented problem is also a solution to the original Stage I problem, although the objective function value of the augmented problem is only an upper bound on the optimal objective function value of the Stage I problem.

The detailed description of the procedure is presented in Algorithm 1. The behavior of this algorithm is controlled through the numbers $\# O U^{\mathrm{opt}}=\left|\mathscr{I}^{\mathrm{opt}}\right|$ and $\# L o c^{\mathrm{opt}}=$ $\left|\mathscr{K}^{\mathrm{opt}}\right|$ of OUs and locations over which we optimize simultaneously within the subproblems. The behavior of the algorithm is also controlled by the stopping conditions. We stopped the algorithm when a time limit of two hours was exceeded or when 100 consecutive iterations of the "while"-loop in Algorithm 1 did not lead to an improved solution. The solution quality and computation times increase as the values of \#OU ${ }^{\text {opt }}$ and $\# L o c^{\text {opt }}$ and the number of repetitions increase. The algorithm was implemented in GAMS 24.0.1, and we used CPLEX 12.5.0.0 to solve the models numerically. We used the CPLEX MipStart feature to start each optimization with the current solution to avoid unnecessary computations. In the CPLEX preprocessing step, a large fraction of the rows and columns of the matrix can typically be eliminated due to constraints (29) and (30). Thus, the small remaining sub-problems can be solved to optimality more rapidly, provided that the numbers $\# O U^{\mathrm{opt}}$ and $\# L o c^{\mathrm{opt}}$ are sufficiently small. The algorithm terminates with a sub-optimal solution of the original problem (without the constraints (29) and (30)).

\section{Case study for a fictitious building based on real-world organizational data}

To evaluate the developed approach, we tested it on the real-world dataset from the MMH, Germany (see Figure 1). In Oucherif (2012), approximately 630,000 records for patient transportation jobs between May 2011 and April 2012 are analyzed to deter- 

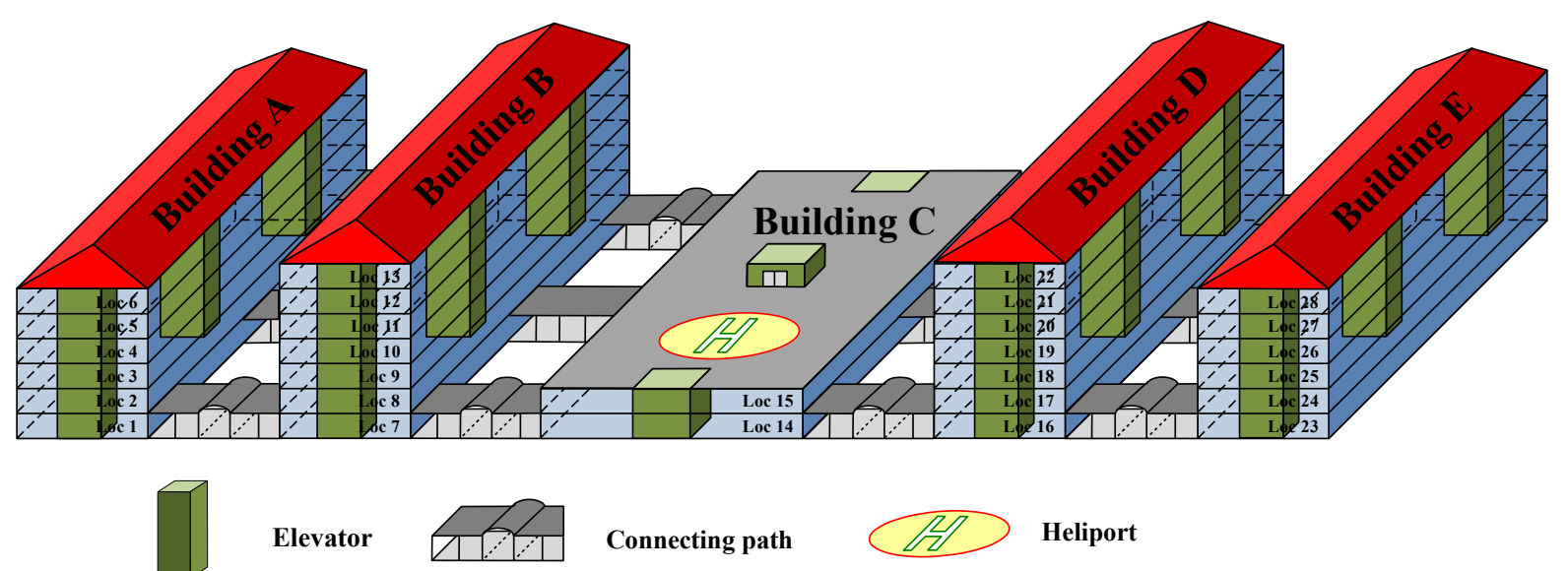

Figure 5: Schematic design of a hypothetical hospital building

Table 3: Aggregated description of the test case

\begin{tabular}{lccc}
\hline Object type & Number & Size range $\left[\mathrm{m}^{2}\right]$ & Total size $\left[\mathrm{m}^{2}\right]$ \\
\hline Wards & 63 & {$[245 ; 1,176]$} & 37,779 \\
Functional units & 42 & {$[49 ; 6,517]$} & 44,051 \\
\hline Locations (Building C) & 2 & 6,762 & 13,524 \\
Locations (other) & 26 & 3,381 & 87,906 \\
\hline
\end{tabular}

mine the number of transports between any two of its OUs. In addition, the current space requirements of those units had been determined. With respect to the locations housing the different OUs, we decided against attempting to hypothetically relocate them within the MHH building structure for several reasons. First, we observed that modern maximum-care hospital buildings tend to be designed in a more compact manner than the one shown in Figure 1 for the MHH. Second, the current layout of the MHH reflects its history of growth and development rather than a coherent design from a logistical perspective. Finally, it would be difficult to divide this aged structure into locations for planning purposes and determine distances in a transparent manner. Thus, we designed the hypothetical hospital building shown in Figure 5. Its 28 locations reflect entire floors of specific buildings. Examples of this type of connected multi-level buildings include the General Hospital in Vienna, Austria, or the university hospital of the Martin-Luther-Universität Halle, Germany.

Table 3 provides the aggregated features of the dataset. The number of OUs (both wards and functional units, such as "Diagnostic imaging" or "Accident and Emergency Department") exceeds the number of locations considerably. Thus, a typical location has to house (elements of) several OUs. However, some OUs are sufficiently large that they must be split up into multiple elements to be allocated to a location. 


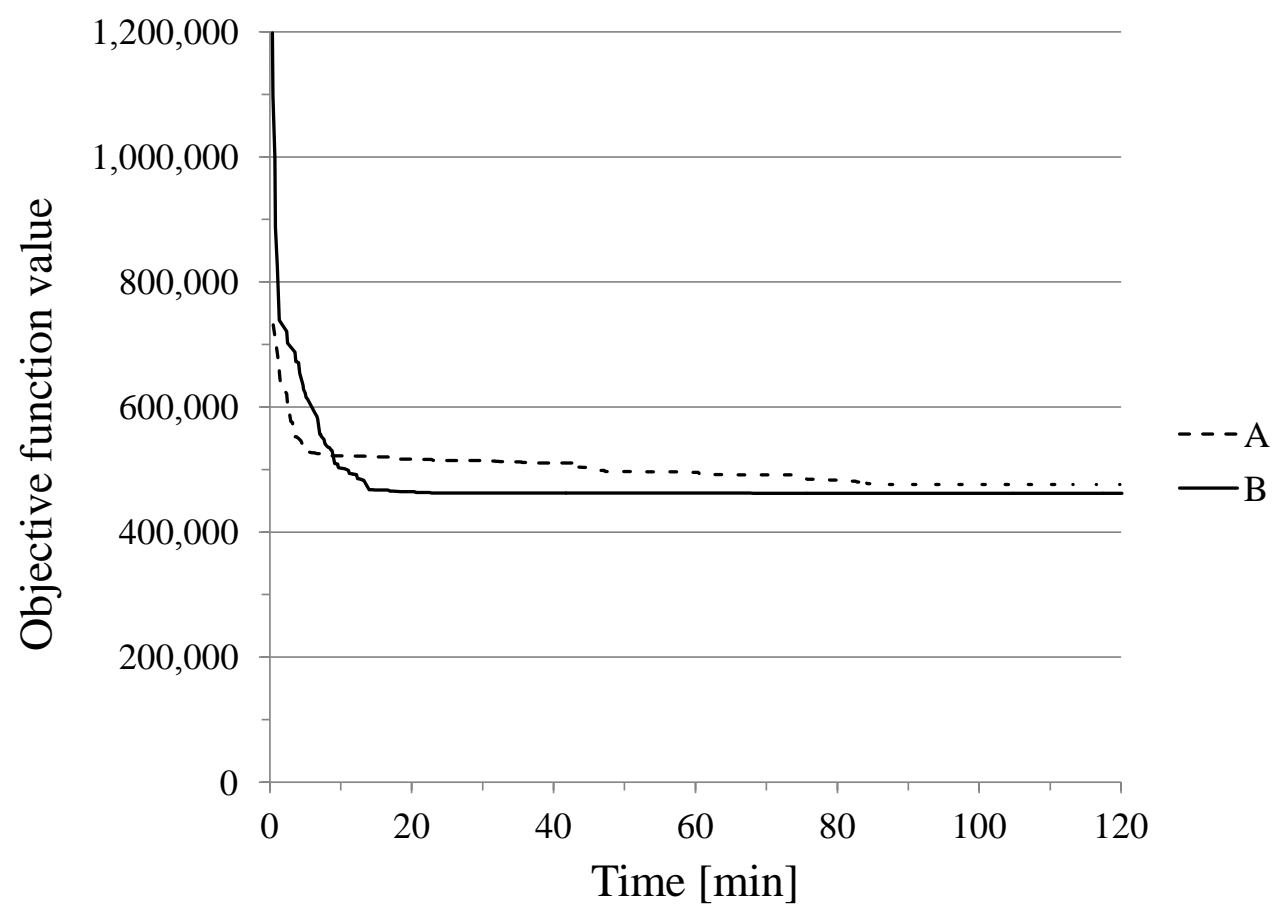

Figure 6: Development of the Stage I objective function value over time

We used an Intel i7-2600 PC with four cores, $3.4 \mathrm{GHz}$, and $16 \mathrm{~GB}$ running under Windows 7, 64 bit, for our computations. The Stage I problem (as formulated in Section 3.3) could not be solved directly for this dataset. Due to the high number of $\widetilde{X}_{\text {iel jfk }}^{I}$ variables, the size of the matrix GAMS provided to CPLEX was in the range of gigabytes, and the initial linear programming relaxation for a branch-and-bound process required approximately $1 \mathrm{~h}$. However, an initial solution could be easily obtained using the approaches explained in Section 4.1, in which we omitted the large number of $\widetilde{X}_{\text {iel jfk }}^{I}$ variables that reflect the QAP aspect of the Stage I problem. The fix-and-optimize heuristic presented in Section 4.2 was successfully able to iteratively improve the initial solutions from either approach A or B in Section 4.1.

Figure 6 presents a typical development of the objective function value as the solution was iteratively improved. The graph illustrates that the idea of approach A to place heavy-traffic OUs close to the center of the hospital leads to a better initial solution than the arbitrary assignment via approach B for this example. However, the solution from approach B outperforms approach A after several iterations. We observed this behavior frequently and conjecture that beginning with an essentially random allocation avoids premature convergence to a local optimum. The graph also illustrates that the difference in the transportation effort between the initial and improved solutions can be substantial. Unfortunately, the optimal objective function value or any other reference 
Table 4: Parameter settings and results for Stage I

\begin{tabular}{|c|c|c|c|c|}
\hline Run & Approach & $\# L o c^{o p t}$ & $\# O U^{o p t}$ & OFV \\
\hline 1 & $\mathrm{~A}$ & 4 & 16 & 492,490 \\
\hline 2 & B & 4 & 16 & 467,857 \\
\hline 3 & A & 6 & 20 & 508,161 \\
\hline 4 & B & 6 & 20 & 492,527 \\
\hline 5 & A & 8 & 24 & 469,230 \\
\hline 6 & B & 8 & 24 & 465,624 \\
\hline 7 & A & 10 & 28 & 475,134 \\
\hline 8 & B & 10 & 28 & 471,731 \\
\hline 9 & A & 12 & 30 & 515,625 \\
\hline 10 & B & 12 & 30 & 491,596 \\
\hline 11 & B & 5 & 10 & 497,096 \\
\hline 12 & B & 3 & 9 & 470,803 \\
\hline 13 & B & 8 & 20 & 467,812 \\
\hline 14 & B & 10 & 20 & 474,644 \\
\hline 15 & B & 8 & 28 & 474,931 \\
\hline 16 & B & 7 & 22 & 466,885 \\
\hline 17 & B & 7 & 24 & 481,094 \\
\hline 18 & B & 4 & 24 & 499,457 \\
\hline 19 & B & 7 & 23 & 479,736 \\
\hline 20 & B & 8 & 26 & 461,946 \\
\hline 21 & A & 8 & 26 & 476,076 \\
\hline
\end{tabular}

methodology with which to compare our results are unavailable.

In Table 4, we report a series of 21 different runs of the fix-and-optimize algorithm for the Stage I problem. We experimented with approaches A and B to determine an initial solution and different numbers of locations and OUs over which to optimize within each of the sub-problems of the fix-and-optimize heuristic. In each case, the total time limit for the Stage I algorithm was set to two hours. The time limit to solve sub-problems to optimality was set to 60 seconds. The results for runs 1 to 10 indicate that the initial solution of approach B consistently outperforms that of approach A. Furthermore, it is sufficient to limit the optimization within each subproblem to a relatively small number of 7 or 8 OUs, provided that the number of locations is relatively large.

We then attempted to solve the Stage II problem using the best solution obtained for the Stage I problem. In the formulation of the model for the Stage II problem, we used the parameter $\alpha$ to balance two competing objectives. One objective was to place the required raster elements for an OU element close to the elevator if the respective OU element will use that elevator often The other objective was to achieve a cohesive assignment of the raster elements. Values of $\alpha$ between 0.0001 and 0.00001 and a 


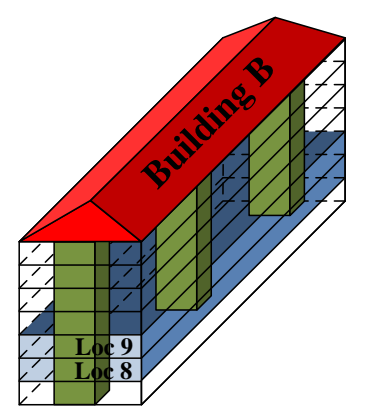

\section{Location 8}

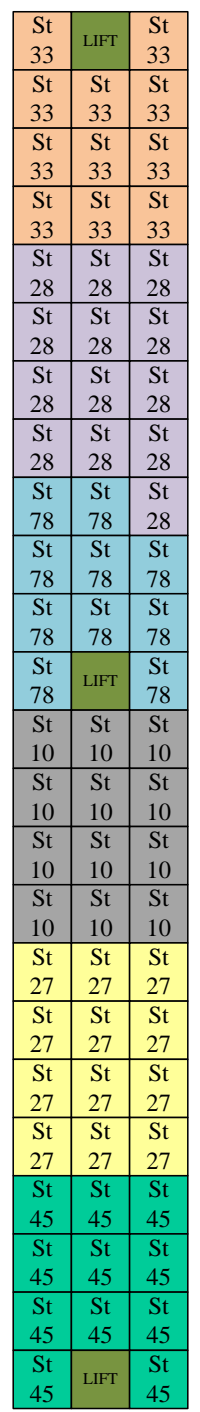

Location 9

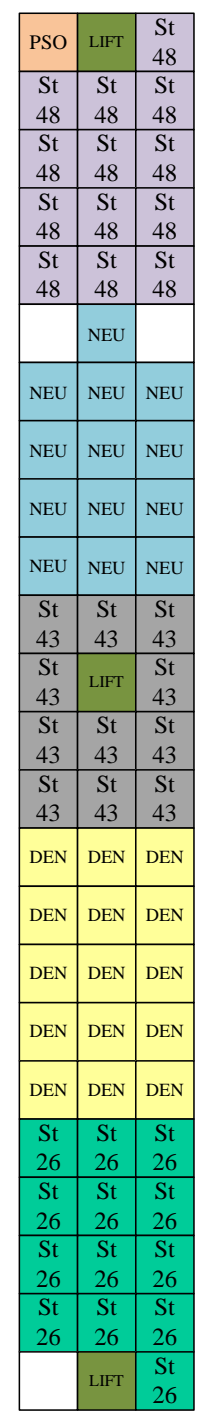

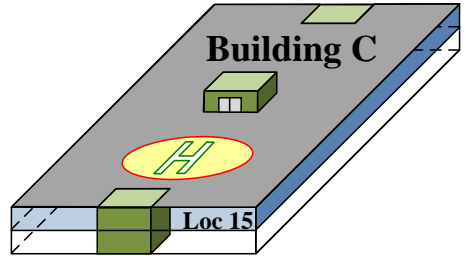

Location 15

\begin{tabular}{|c|c|c|c|c|c|}
\hline OP & $\mathrm{OP}$ & LIFT & LIFT & OP & OP \\
\hline OP & $\mathrm{OP}$ & OP & $\mathrm{OP}$ & OP & $\mathrm{OP}$ \\
\hline $\mathrm{OP}$ & OP & OP & OP & OP & $\mathrm{OP}$ \\
\hline OP & $\mathrm{OP}$ & OP & $\mathrm{OP}$ & OP & OP \\
\hline OP & OP & OP & $\mathrm{OP}$ & OP & $\mathrm{OP}$ \\
\hline OP & OP & OP & OP & OP & OP \\
\hline OP & OP & OP & OP & OP & OP \\
\hline OP & OP & OP & OP & OP & OP \\
\hline OP & OP & OP & OP & OP & OP \\
\hline OP & $\mathrm{OP}$ & $\mathrm{OP}$ & OP & OP & OP \\
\hline OP & $\mathrm{OP}$ & $\mathrm{OP}$ & OP & OP & $\mathrm{OP}$ \\
\hline $\begin{array}{l}\text { St } \\
85\end{array}$ & $\begin{array}{l}\text { St } \\
85\end{array}$ & LIFT & LIFT & $\begin{array}{l}\mathrm{St} \\
46\end{array}$ & $\begin{array}{l}\mathrm{St} \\
46\end{array}$ \\
\hline $\mathrm{St}$ & St & $\mathrm{St}$ & St & St & $\mathrm{St}$ \\
\hline 85 & 85 & 85 & 46 & 46 & 46 \\
\hline $\mathrm{St}$ & $\mathrm{St}$ & St & $\mathrm{St}$ & $\mathrm{St}$ & $\mathrm{St}$ \\
\hline 85 & 85 & 85 & 46 & 46 & 46 \\
\hline $\mathrm{St}$ & $\mathrm{St}$ & $\overline{\mathrm{St}}$ & $\mathrm{St}$ & $\mathrm{St}$ & $\mathrm{St}$ \\
\hline 85 & 85 & 85 & 46 & 46 & 46 \\
\hline $\begin{array}{l}\mathrm{St} \\
85\end{array}$ & $\begin{array}{l}\text { St } \\
85\end{array}$ & BLB & BLB & BLB & BLB \\
\hline BLB & BLB & BLB & BLB & BLB & BLB \\
\hline BLB & BLB & BLB & BLB & BLB & BLB \\
\hline BLB & BLB & BLB & BLB & BLB & BLB \\
\hline $\begin{array}{l}\mathrm{St} \\
86\end{array}$ & BLB & BLB & BLB & BLB & BLB \\
\hline $\begin{array}{l}\mathrm{St} \\
86\end{array}$ & $\begin{array}{l}\text { St } \\
86\end{array}$ & BLB & BLB & BLB & BLB \\
\hline $\begin{array}{l}\mathrm{St} \\
86\end{array}$ & $\begin{array}{l}\text { St } \\
86\end{array}$ & $\begin{array}{l}\text { St } \\
86\end{array}$ & BLB & BLB & BLB \\
\hline $\begin{array}{l}\text { St } \\
86\end{array}$ & $\begin{array}{l}\text { St } \\
86\end{array}$ & $\begin{array}{l}\text { St } \\
86\end{array}$ & BLB & BLB & BLB \\
\hline $\begin{array}{l}\mathrm{St} \\
86\end{array}$ & $\begin{array}{l}\text { St } \\
86\end{array}$ & LIFT & LIFT & BLB & BLB \\
\hline
\end{tabular}

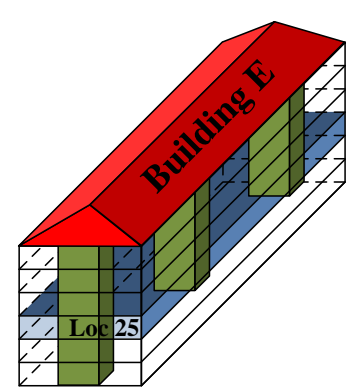

Location 25

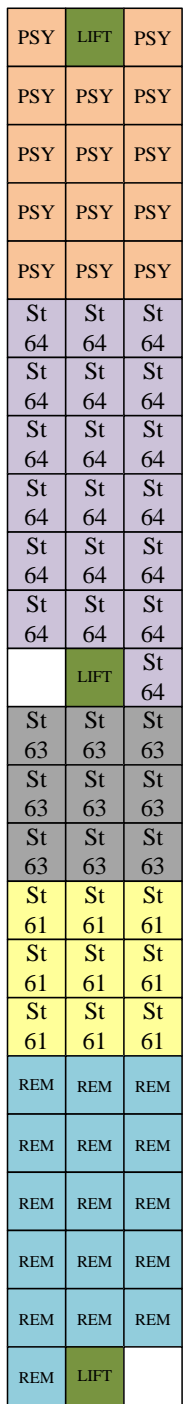

Figure 7: Selection of results for Stage II

computation time limit of 1,800 seconds were required to achieve adequate and largely cohesive solutions.

In Figure 7, we present a selection of results for four out of the 28 different locations of our hypothetical hospital. The results are driven by a logistical perspective on 


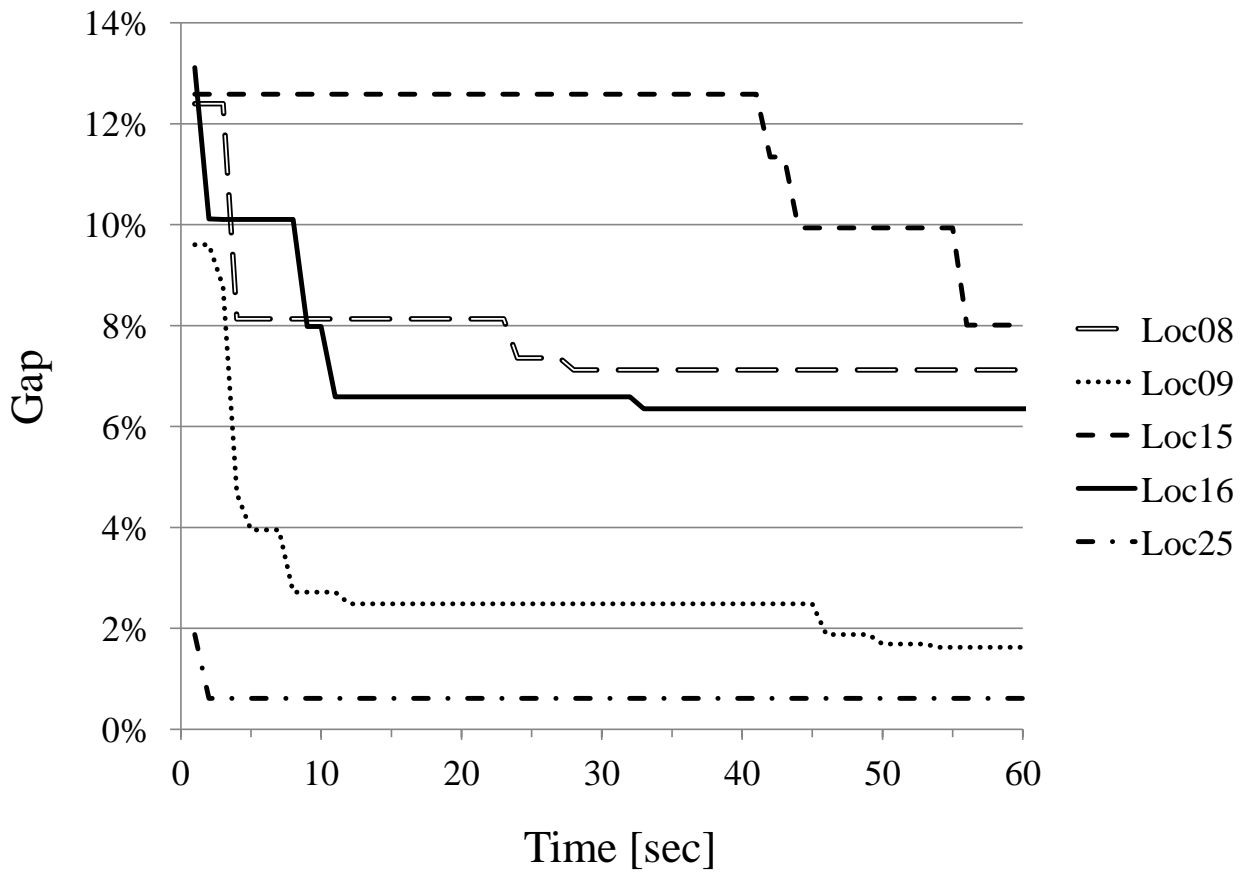

Figure 8: Development of the optimality gap for selected Stage II models

the problem. This perspective suggests a structure in which wards (indicated by station numbers) and functional units (indicated by abbreviations) are mixed within the locations. If such a structure is not desired, it can easily be avoided by adjusting the set $\mathscr{K}_{i}$ of those locations, to which the elements of an OU $i$ can be assigned at all.

Figure 8 illustrates how the optimality gap for selected Stage II models evolved over time during the first 60 seconds of the optimization. Whereas models for certain locations could be solved to optimality rather quickly, others were more difficult to solve. We stopped the optimization for a Stage II problem of a single location when a time limit of 1,800 seconds was reached without finding an optimal solution. The output of the Stage II models must be considered as a starting point for a manual fine-tuning process.

The most important output of the proposed planning system is the solution to the Stage I problem. The results presented in Figure 6 and Table 4 clearly indicate that due to the highly inhomogeneous traffic between the different OUs of a maximumcare hospital, the layout can have a tremendous impact on the resources required for transportation processes. Establishing an efficient layout is clearly a non-trivial task. The modeling system presented here can support a planner facing that task. 


\section{Conclusion}

We have analyzed and modeled the problem of assigning the different OUs of a large maximum-care hospital within a given building structure that is sufficiently large to host those units. We considered the space requirements and other real-world aspects with the aim of obtaining a solution for the layout problem that minimizes the transportation effort. To this end, we developed a hierarchical modeling system and a decomposition algorithm that enabled us to solve problem instances with real-world characteristics despite the computationally challenging underlying QAP. The computational burden of the approach appears to be acceptable given the enormous planning effort to design such a large maximum-care hospital. Although the proposed approach is neither intended nor suited to fully automate the process of generating a solution for the layout problem, it can systematically support this process.

\section{References}

Adams, W. P. and H. D. Sherali (1986). A Tight Linearization and an Algorithm for Zero-One Quadratic Programming Problems. Management Science 32(10), 1274-1290.

Armour, G. C. and E. S. Buffa (1963). A Heuristic Algorithm and Simulation Approach to Relative Location of Facilities. Management Science 9(2), 294-309.

Barbosa-Póvoa, A. P., R. Mateus, and A. Q. Novais (2001). Optimal two-dimensional layout of industrial facilities. International Journal of Production Research 39(12), 2567-2593.

Barbosa-Póvoa, A. P., R. Mateus, and A. Q. Novais (2002). Optimal 3D layout of industrial facilities. International Journal of Production Research 40(7), 1669-1698.

Böhme, D. (2013). Entwicklung von Entscheidungsmodellen für die innerbetriebliche Standortplanung von Akutkrankenhäusern. Gottfried Wilhelm Leibniz Universität Hannover: Masterarbeit, Institut für Produktionswirtschaft.

Bozer, Y. A. and R. D. Meller (1997). A reexamination of the distance-based facility layout problem. IIE Transactions 29(7), 549-560.

Bozer, Y. A., R. D. Meller, and S. Erlebacher (1994). An Improvement-Type Layout Algorithm for Single and Multiple-Floor Facilities. Management Science 40(7), 918-932.

Burkard, R. E., S. Karisch, and F. Rendl (1991). QAPLIB-A quadratic assignment problem library. European Journal of Operational Research 55(1), 115-119.

Burkard, R. E. and J. Offermann (1977). Entwurf von Schreibmaschinentastaturen mittels quadratischer Zuordnungsprobleme. Zeitschrift für Operations Research 21(4), 121-132.

Butler, T. W., K. R. Karwan, J. R. Sweigart, and G. R. Reeves (1992). An Integrative Model-Based Approach to Hospital Layout. IIE Transactions 24(2), 144-152. 
Drira, A., H. Pierreval, and S. Hajri-Gabouj (2007). Facility layout problems: A survey. Annual Reviews in Control 31(2), 255-267.

Elshafei, A. N. (1977). Hospital Layout as a Quadratic Assignment Problem. Operational Research Quarterly 28(1), 167-179.

Foulds, L. R. (1983). Techniques for Facilities Layout: Deciding Which Pairs of Activities Should be Adjacent. Management Science 29(12), 1414-1426.

Garey, M. R. and D. S. Johnson (2009). Computers and intractability: A guide to the theory of NP-completeness (27. print Aufl.). A series of books in the mathematical sciences. New York NY u.a: Freeman.

Glover, F. and E. Woolsey (1974). Converting the 0-1 Polynomial Programming Problem to a 0-1 Linear Program. Operations Research 22(1), 180-182.

Hahn, P., J. MacGregor Smith, and Y.-R. Zhu (2010). The Multi-Story Space Assignment Problem. Annals of Operations Research 179(1), 77-103.

Hahn, P. M. and J. Krarup (2001). A hospital facility layout problem finally solved. Journal of Intelligent Manufacturing 12, 487-496.

Hahn, P. M., Y.-R. Zhu, M. Guignard, W. L. Hightower, and M. J. Saltzman (2012). A Level-3 Reformulation-Linearization Technique-Based Bound for the Quadratic Assignment Problem. INFORMS Journal on Computing 24(2), 202-209.

Hassan, M. M. D. (1994). Machine layout problem in modern manufacturing facilities. International Journal of Production Research 32(11), 2559-2584.

Helber, S. and F. Sahling (2010). A fix-and-optimize approach for the multi-level capacitated lot sizing problem. International Journal of Production Economics 123(2), 247-256.

Heragu, S. S. and A. Kusiak (1991). Efficient models for the facility layout problem. European Journal of Operational Research 53(1), 1-13.

Hillier, F. S. and M. M. Connors (1966). Quadratic Assignment Problem Algorithms and the Location of Indivisible Facilities. Management Science 13(1), 42-57.

Koopmans, T. C. and M. Beckmann (1957). Assignment Problems and the Location of Economic Activities. Econometrica 25(1), 53-76.

Kusiak, A. and S. S. Heragu (1987). The facility layout problem. European Journal of Operational Research 29(3), 229-251.

Levary, R. R. and S. Kalchik (1985). Facilities layout - a survey of solution procedures. Computers \& Industrial Engineering 9(2), 141-148.

Loiola, E. M., N. M. M. de Abreu, P. O. Boaventura-Netto, P. Hahn, and T. Querido (2007). A survey for the quadratic assignment problem. European Journal of Operational Research 176(2), $657-690$.

Oral, M. and O. Kettani (1992). A Linearization Procedure for Quadratic and Cubic MixedInteger Problems. Operations Research 40, 109-116. 
Oucherif, F. (2012). Analyse und Optimierung der Layoutplanung von Krankenhäusern aus Sicht der Transportlogistik durch Methoden des Operations Research. Gottfried Wilhelm Leibniz Universität Hannover: Masterarbeit, Institut für Produktionswirtschaft.

Sahling, F. (2010). Mehrstufige Losgrößenplanung bei Kapazitätsrestriktionen. Gabler Research : Produktion und Logistik. Wiesbaden: Gabler.

Steinberg, L. (1961). The Backboard Wiring Problem: A Placement Algorithm. SIAM Review 3(1), 37-50.

Vos, L., S. Groothuis, and G. G. van Merode (2007). Evaluating hospital design from an operations management perspective. Health Care Management Science 10(4), 357-364. 